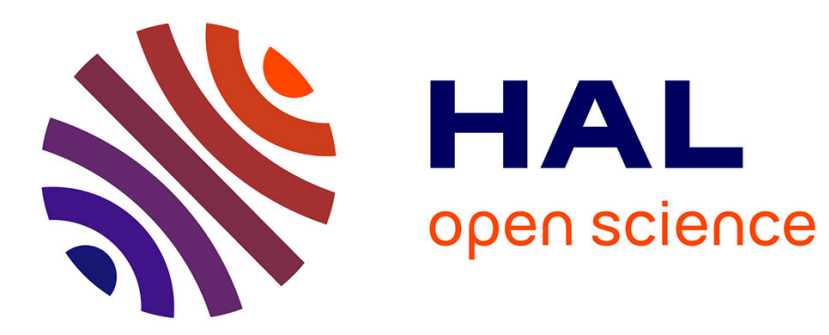

\title{
Deux nouvelles espèces de crustacés décapodes de l'Albien du Bassin de Paris
}

Gérard Breton

\section{To cite this version:}

Gérard Breton. Deux nouvelles espèces de crustacés décapodes de l'Albien du Bassin de Paris. Geodiversitas, 2013, 33 (2), pp.279-284. 10.5252/g2011n2a5 . insu-00966708

\section{HAL Id: insu-00966708 https://hal-insu.archives-ouvertes.fr/insu-00966708}

Submitted on 3 Mar 2021

HAL is a multi-disciplinary open access archive for the deposit and dissemination of scientific research documents, whether they are published or not. The documents may come from teaching and research institutions in France or abroad, or from public or private research centers.
L'archive ouverte pluridisciplinaire HAL, est destinée au dépôt et à la diffusion de documents scientifiques de niveau recherche, publiés ou non, émanant des établissements d'enseignement et de recherche français ou étrangers, des laboratoires publics ou privés. 


\title{
Deux nouvelles espèces de crustacés décapodes de l'Albien du Bassin de Paris
}

\author{
Gérard BRETON \\ Laboratoire Géosciences, Université de Rennes 1, \\ F-35042 Rennes cedex (France) \\ et 6 rue des Réservoirs, \\ F-76600 Le Havre (France) \\ gerardbreton@free.fr
}

MOTS CLÉS

Crustacea,

Decapoda, nomenclature, Albien,

Bassin de Paris, espèces nouvelles.

KEY WORDS

Crustacea,

Decapoda, nomenclature, Albian,

Paris Basin, new species.
Breton G. 2011. - Deux nouvelles espèces de crustacés décapodes de l'Albien du Bassin de Paris. Geodiversitas 33 (2): 279-284. DOI: 10.5252/g2011n2a5.

\section{RÉSUMÉ}

Deux nouvelles espèces de crustacés décapodes, Glyphea franzini n. sp. (Glypheidae) et Callianassa agae n. sp. (Callianassidae), du faciès Gault de l'Albien du Bassin de Paris, sont décrites ici. Ces deux espèces ont été précédemment décrites et figurées par Breton (2010) dans le CD-Rom d'un ouvrage collectif de grande diffusion intitulé Stratotype Albien. Néanmoins, conformément à l'article $8.6 \mathrm{du}$ Code international de nomenclature zoologique concernant les travaux produits après 1999 par un procédé sans support papier, ces deux noms ne sont pas disponibles. En effet, le CD-Rom ne contient pas de déclaration de l'auteur selon laquelle des copies ont été déposées dans au moins cinq grandes bibliothèques publiques, ces bibliothèques devant être énumérées dans le travail lui-même.

\section{ABSTRACT}

Two new species of decapod crustaceans from the Albian of the Paris Basin. Two new species of decapod crustaceans, Glyphea franzini n. sp. (Glypheidae), and Callianassa agae n. sp. (Callianassidae), from the Albian Gault facies of the Paris Basin, are described herein. These two species were previously described and figured by Breton (2010) in the CD-Rom of a popularization collective book entitled Stratotype Albien. Nevertheless, in respect to the article 8.6 of the International Code of Zoological Nomenclature concerning the works produced after 1999 by a method that does not employ printing on paper, these two names are not available. Indeed, the CD-Rom does not contain a statement that copies have been deposited at least in five major publicly accessible libraries which should be identified by name in the work itself. 


\section{INTRODUCTION}

Les descriptions de deux espèces nouvelles de crustacés décapodes qui figurent dans un ouvrage collectif de grande diffusion (Breton 2010b) ne satisfont pas à l'article $8.6 \mathrm{du}$ Code international de nomenclature zoologique (CINZ 1999). Ces espèces on été décrites dans un CD-Rom édité en même temps que l'ouvrage; leur description ne figure pas sur l'édition papier de l'ouvrage. De plus, ce CD-Rom ne répond pas aux prescriptions précises de l'article 8.6 puisqu'il n'y figure pas la liste des cinq grandes bibliothèques publiques où le $\mathrm{CD}$-Rom est déposé. Une nouvelle description est donc maintenant présentée ici, sur un support papier, afin de rendre les noms disponibles.

\section{GISEMENTS}

Les spécimens proviennent de deux gisements. Dans l'ancienne carrière Ledoigt, à Bully (Seine-Maritime), route de Saint-Martin-l'Hortier, affleuraient des argiles de faciès Gault, de l'Albien inférieur et moyen, niveau a (biozone à Bulliensis) à h (biozone à Steinmanni) (Destombes et al. 1977). Dans la carrière de Pargnysur-Saulx (Marne), exploitée par Yméris-Toiture, affleurent des argiles de l'Albien inférieur et moyen, Formation des Argiles tégulines de Courcelles, biozone à Steinmanni, à sous-biozone à Pseudolyelli.

\section{LIEUX DE CONSERVATION DES SPÉCIMENS}

Les spécimens sont conservés dans la collection de Paléontologie du Muséum national d'Histoire naturelle, Paris (MNHN.F) et dans celle du Muséum d'Histoire naturelle du Havre (MHNH).

\section{SYSTÉMATIQUE}

Classe MALACOSTRACA Latreille, 1802 Ordre DECAPODA Latreille, 1802 Infra-ordre GLYPHEIDEA Winckler, 1882 Famille GLYPHEIDAE Winckler, 1882

$$
\text { Genre Glyphea Von Meyer, } 1835
$$

ESPÈCE TYPE. - Palinurus regleyanus Desmarets, 1822 par désignation originale.

\section{Glyphea franzini n. sp.}

(Fig. 1)

Glyphea franzini Breton, 2010a: 224, fig. 149H; 230, fig. $155 \mathrm{~A}, \mathrm{~B}$ (version papier); 2010b: 10-13, figs $1 \mathrm{H}$, 7 (Cd-rom). Non disponible en application de l'article $8.6 \mathrm{du}$ Code.

MATÉRIEL TYPE. - Holotype (MNHN.F.A28980, ex coll. Franzin; Fig. 1), figuré par Breton (2010a: figs 149H, 155A; 2010b: figs $1 \mathrm{H}, 7 \mathrm{~A}$ ); paratype (MNHN.F.A28981, coll. Franzin), figuré par Breton (2010a: fig. 155B; 2010b: figs $1 \mathrm{H}, 7 \mathrm{~B})$.

LOCALITÉ TYPE. - Carrière de Pargny-sur-Saulx (Marne, France); Formation des Argiles tégulines de Courcelles, faciès Gault, Albien inférieur-moyen.

DIMENSIONS DES TYPES. - Holotype: longueur conservée de la carapace: $13 \mathrm{~mm}$, longueur estimée: 14 à $15 \mathrm{~mm}$; hauteur: 6,5 mm; largeur: 3,1 mm. Le paratype est un peu plus grand (longueur conservée: 18,1 mm).

ÉTYMOLOGIE. - L'espèce est dédiée au paléontologue Benjamin Franzin, qui a recueilli le matériel type et nous l'a communiqué.

Distribution. - Albien, faciès Gault de l'Est du Bassin parisien: Pargny-sur-Saulx (Marne).

DiAGNOSE. - Carapace comprimée latéralement, plus de deux fois plus longue que haute. Carène médiodorsale flanquée d'une double rangée de tubercules épineux pointant vers l'avant. Sillon cervical profond, rectiligne, incliné à $75^{\circ}$ par rapport à la marge dorsale. Sillon antennaire très court, sillon hépatique large, droit, court, joignant l'extrémité inférieure du sillon cervical à celle du sillon post-cervical. Sillon post-cervical dessinant un grand virage avant un trajet postérieur rectiligne et presque horizontal; il s'élargit, s'estompe et perd son individualité vers l'arrière. Sillon branchiocardiaque parallèle au sillon post-cervical, plus étroit et plus marqué que ce dernier. La partie antérieure du céphalothorax porte trois carènes parallèles longitudinales, munies de tubercules épineux pointant vers l'avant et disposés en dents de scie. La surface entre les carènes est concave et lisse à l'exception: 1) d'une rangée de tubercules entre les carènes médiane et ventrale; et 2) d'un groupe d'une dizaine de tubercules sous la carène ventrale. La carène dorsale, proche de la ligne médiane participe à la double rangée de tubercules épineux pointant vers l'avant qui flanque la carène longitudinale médiane dorsale. Un alignement de tubercules flanque postérieurement le sillon cervical; une demi-douzaine de tubercules forment un arc de cercle au-dessus du sillon hépatique. Le reste de la carapace est couvert de gros tubercules épineux projetés vers le dos et vers 


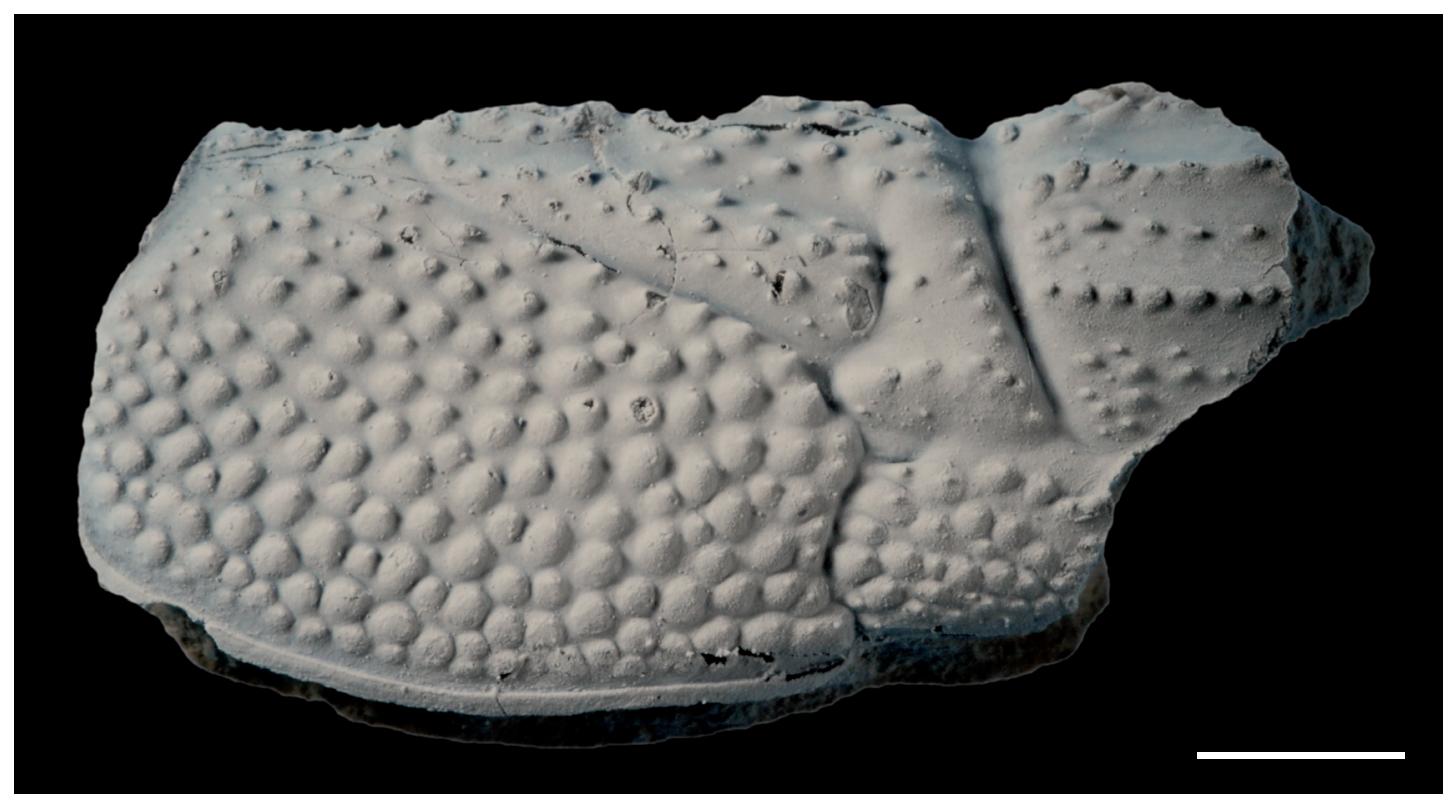

FIG. 1. - Glyphea franzini n. sp., holotype (MNHN.F.A28980), céphalothorax blanchi au chlorure d'ammonium sublimé, vue latérale droite, Pargny-sur-Saulx (Marne), Albien inférieur. Échelle: $2 \mathrm{~mm}$.

l'avant et dont la taille décroît progressivement vers le dos et vers l'arrière. Le bord postérieur, arrondi, et le bord ventral sont jalonnés par un sillon.

\section{REMARQUES}

La double rangée médio-dorsale de tubercules épineux pointant antérieurement n'est bien visible que sur le paratype, par ailleurs moins bien conservé et moins bien dégagé que l'holotype. La partie antérieure n'est pas complète, la région rostrale manque sur l'un et l'autre spécimens. Appendices et abdomen ne sont pas conservés dans le matériel étudié ici.

\section{DIFFÉRENCES AVEC LES ESPÈCES VOISINES}

Glyphea cretacea McCoy, 1854 a des proportions différentes, un sillon cervical moins rectiligne et n'a pas de tubercules entre les carènes moyenne et ventrale.

Glyphea carteri Bell, 1862 a une ornementation plus proche de celle de $G$. franzini n. sp., dont elle se distingue par les proportions des régions et la disposition des sillons.
Le trajet et la disposition des sillons de $G$. willetti (Woodward, 1878) sont distincts de ceux de G. franzini n. sp., de plus la marge postérieure est sinueuse. La double rangée de tubercules jalonnant la carène médiane dorsale est un trait partagé avec $G$. willetti, que l'on ne trouve pas dans les deux autres espèces.

Glyphea franzini n. sp. differe de G. foresti Feldmann \& de Saint-Laurent, 2002 du Cénomanien d'Australie par sa plus petite taille, son céphalothorax plus haut, son ornementation beaucoup plus vigoureuse, et par un sillon antennaire bien moins marqué. De plus, ses carènes céphaliques sont tuberculées, même dans leur partie postérieure, et la région entre les sillons branchiocardiaque et cervical porte des tubercules.

Glyphea franzini n. sp. ressemble par plusieurs traits morphologiques à l'espèce de l'Albien supérieur de Pologne et d'Angleterre Glypheopsis sanctaecrucis Collins, 1969 (= Glyphea sanctaecrucis, les différences entre le genre Glypheopsis Beurlen, 1928 créé par Beurlen [1928] pour des espèces jurassiques et le genre Glyphea von Meyer, 1835 n'étant pas suffisantes à notre avis pour valider une distinction 
générique). Elles ont en commun la petite taille, la double rangée de tubercules le long de la ligne médio-dorsale, l'alignement de petits granules entre la seconde et la troisième carène longitudinale antérieure, et le groupe de fins granules entre la troisième carène et la marge ventrale. Les caractères suivants de $G$. franzini n. sp. permettront de la différencier de $G$. sanctaecrucis: sillon postcervical ne rejoignant pas le sillon branchiocardiaque, et n'atteignant pas la ligne médio-dorsale; absence des fins sillons courts émanant du sillon postcervical; ornementation de la région branchiale plus grossière, formée de tubercules de taille inégale; les deux lignes de tubercules de part et d'autre de la suture médio-dorsale distincts jusqu’à la marge postérieure; marge postérieure du céphalothorax moins inclinée de profil.

\section{Infra-ordre AXIIDEA de Saint-Laurent, 1979 Famille Callianassidae Dana, 1852}

\section{Genre Callianassa Leach, 1814}

ESPÈCE TYPE. - Astacus subterraneus Montagu, 1808, par désignation originale.

\section{Callianassa agae n. $\mathrm{sp}$.}

(Fig. 2)

Callianassa sp. - Collins in Hancock et al. 1965: 254.

Callianassa agae Breton, 2010a: 225, fig. 150A (version papier); 2010b: 15, fig. 2A (CdRom). Non disponible en application de l'article $8.6 \mathrm{du}$ Code.

Matériel type. - Holotype (propode, MNHN.F.A28983, coll. Breton, Fig. 2A); paratype 1 (propode, MNHN.F.A28984, coll. Breton, Fig. 2B); paratype 2 (propode, MNHN.F.A28985, coll. Breton); paratype 3 (propode, MNHN.F.A28986, coll. Breton, Fig. 2C) ; paratype 4 (propode, MNHN.F.A28987, coll. Breton); paratype 5 (propode, MNHN.F.A28988, coll. Breton); paratype 6 (propode, MNHN.F.A28989, coll. Breton); paratype 7 (carpe, MNHN.F.A28990, coll. Breton). Holotype et paratypes 1-6: ex coll. D. Portail, 1980; paratypes 7, 8: ex coll. G. Breton, 16.VII.2006.

LOCALITÉ TYPE. - Bully (zone à Bulliensis, niveau a 1-2), Aube, France.
DIMENSIONS DE L'HOLOTYPE. — Longueur totale conservée du propode: $15,1 \mathrm{~mm}$; longueur totale estimée du propode: $17,5 \mathrm{~mm}$; longueur de la paume: $10,0 \mathrm{~mm}$; largeur du propode: $8,8 \mathrm{~mm}$; épaisseur maximum du propode: $4,0 \mathrm{~mm}$; longueur estimée du doigt fixe: $7,6 \mathrm{~mm}$ largeur de la base du doigt fixe: $1,9 \mathrm{~mm}$; épaisseur du doigt fixe: 1,9 $\mathrm{mm}$. Mesures effectuées au micromètre oculaire sous un stéréomicroscope.

ORIGINE DU MATÉRIEL TYPE. - Bully (zone à Bulliensis, niveau a 1-2): holotype, paratypes 1, 2; Bully (zone à Normanniae, niveau d (=R2, Destombes 1973): paratypes 3-5; Pargny-sur-Saulx: paratypes 6, 7.

Autre Matériel obSerVÉ. - Spécimens collectés par Franzin, Matrion, Touch, Colleté, Fricot, Breton, Portail, Destombes et provenant des gisements de l'Aube, de la Marne, de la Seine-Maritime et du Pas-de-Calais; spécimens du Gault d'Angleterre. Remarque: le spécimen MHNH 7876 de Bully est une composition habilement faite à partir de deux propodes donnant l'illusion d'un propode en connexion anatomique avec un carpe.

ÉTYMOLOGIE. - Le nom d'espèce est formé sur l'acronyme de l'Association géologique auboise, à qui l'espèce est dédiée, pour rendre hommage à ses remarquables travaux de recherche et de vulgarisation sur la géologie en particulier de l'Aube et de l'Albien.

Distribution. - Faciès argileux de l'Albien (Gault) du Bassin anglo-parisien.

DiAGNOSE. — Paume (= propode sans le doigt fixe) presque carrée, comprimée, bords supérieur et inférieur droits, parallèles, soulignés par une arête continue, perpendiculaires aux bords distal et proximal. Face externe régulièrement convexe, face interne convexe au centre mais deux concavités longitudinales jouxtent les arêtes, la concavité inférieure moins marquée. Longueur du doigt fixe 70 à $80 \%$ de la longueur de la paume. Doigt fixe droit, dans le prolongement de l'arête inférieure, à peine recourbé vers l'intérieur à son extrémité, à section rectangulaire, face regardant vers le dactyle plate, portant sur son bord externe une rangée de tubercules, très petits à la base et au bout du doigt, plus gros au milieu. Juste à l'extérieur de cette rangée de tubercules, ainsi qu'à l'intérieur immédiat des arêtes inférieure et supérieure de la paume, se prolongeant jusqu'au bout du doigt fixe pour l'arête inférieure, sont alignés de petits trous dans lesquels s'insérait vraisemblablement une rangée de soies. Dactyle droit, aussi long que le doigt fixe. Articulation avec le carpe ovale, occupant un peu moins de la moitié de la largeur du propode. Articulation avec le dactyle très large, occupant un peu moins des $2 / 3$ de la largeur du propode. Carpe rarement conservé, un peu plus long que large. Ornementations formées de surépaisseurs de la cuticule, plus foncées, en flammes ou "en atolls» (Breton et al. 2003) étirées ou alignées transversalement. 


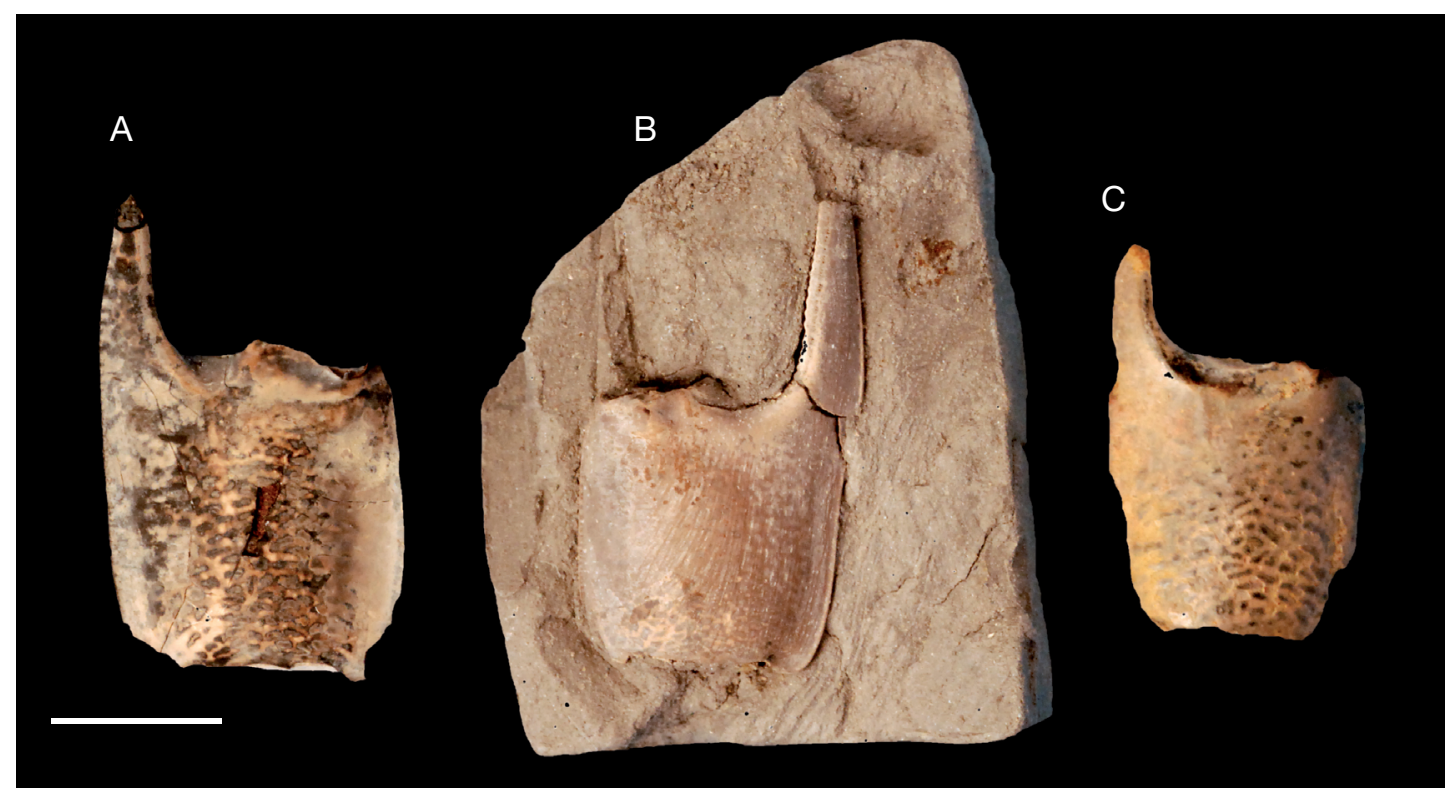

FIG. 2. - Propodes de Callianassa agae n. sp. de Bully (Seine-Maritime), Albien inférieur: A, holotype (MNHN.F.A28983, zone à Bulliensis, niveau a1-2), face interne; B, paratype 1 (MNHN.F.A28984, zone à Bulliensis, niveau a1-2), face externe; C, paratype 3 (MNHN.F.A28986, zone à Normanniae, niveau d), face interne. Échelle: $5 \mathrm{~mm}$.

\section{PRÉSERVATION}

La cuticule est conservée (holotype, paratypes 1, 2, 6, 7) ou non (paratypes 3-5). Ces derniers proviennent d'un horizon où les fossiles sont remaniés à l'état de moules internes phosphatés. Dans ce cas, l'ornementation, les tubercules et les trous d'insertion de soies ne sont pas visibles. Le dactyle, dans notre matériel, n'est connu que par une empreinte (paratype 1). Le doigt fixe est très fréquemment cassé. Au sein des séries examinées, les carpes sont étonnamment rares et mal conservés, probablement parce que leur cuticule est plus fragile que celle des propodes. Quelques nodules montrent, associés à des propodes, d'autres appendices et des fragments très mal conservés de l'abdomen et peut-être du céphalothorax. Leur cuticule est mince, brune, brillante, non ornementée. La morphologie des propodes est très homogène, s'il y a une hétérochélie, elle est uniquement dimensionnelle.

\section{RAPPORT ET DIFFÉRENCES}

AVEC LES ESPÈCES VOISINES

Callianassa neocomiensis Woodward, 1868 est une espèce de l'Upper Greensand d'Angleterre, beaucoup plus grande que C. agae n. sp. (28 mm), et qui en differe par les proportions du propode. Joe Collins (in litt.) précise qu'il est probable que c'est par confusion, sous le nom de $C$. neocomiensis, que l'espèce du Gault, $C$. agae n. sp., a pu être citée occasionnellement. Callianassa cenomaniensis A. Milne-Edwards, 1861 a des propodes plus allongés, plus arrondis, l'articulation carpe-propode est plus oblique, et c'est ce critère qui conduit Mertin (1941) à classer cette espèce dans le genre Protocallianassa, et à en faire une sous-espèce de $P$. antiqua Roemer, 1841, mais cette dernière espèce semble avoir une articulation carpepropode perpendiculaire ou peu oblique. Callianassa antiqua Roemer, 1841 a des propodes plus allongés et des doigts plus courts, plus trapus.

\section{Remerciements}

Je remercie chaleureusement Philippe Bouchet et Didier Merle (Muséum national d'Histoire naturelle, Paris) qui ont attiré mon attention sur le problème nomenclatural posé et qui ont proposé une solution. 


\section{RÉFÉRENCES}

BeuRlen K. 1928. — Die Decapoden des Schwabischen Jura mit Ausnahme der aus den oberjurassichen Plattenkalken Stammenden. Palaeontographica 70: 115-278.

Breton G. 2010a. - Les crustacés, in Colleté C. (ed.), Stratotype Albien. Muséum national d'Histoire naturelle, Paris; Biotope, Mèze; BRGM, Orléans, Patrimoine géologique (2), 221-230 p.

Breton G. 2010b. - Les crustacés, in Colleté C. (ed.), Stratotype Albien. Muséum national d'Histoire naturelle, Paris; Biotope, Mèze; BRGM, Orléans, Patrimoine géologique (2), Cd-Rom: 3-31.

Breton G., Carpentier C., Huault V. \& Lathuilière B. 2003. - Decapod crustaceans from the Kimmeridgian of Bure (Meuse, France). Contributions to Zoology 72 (2-3): 91-93.

Collins J. S. H. 1965. — Decapod and Cirripede Crustacea, in Hancock J. M. et al., The Gault of the Weald. Pro- ceedings of the Geologists' Association 76 (3): 252-255.

COMMISSION INTERNATIONALE DE NOMENCLATURE ZOOLOGIQUe (CINZ) 1999. - Code international de nomenclature zoologique. $4 \mathrm{e}$ éd. International Trust for Zoological Nomenclature, c/o The Natural History Museum, Londres: xxix $+306 \mathrm{p}$.

Destombes P. 1973. - Hoplitidae et zonation nouvelle de l'Albien inférieur de Bully-Saint-Martin (Bray occidental). Comptes rendus de l'Académie des Sciences, Paris 277: 2145-2148.

Destombes P., Gamble H. J., Juignert P. \& Owen H. G. 1977. - Cretaceous and lower Tertiary of SeineMaritime, France: a guide to key localities. Proceedings of the Geologists' Association 88 (1): 15-38.

MERTIN H. 1941. - Decapode Krebse aus dem subhercynen und Braunschweiger Emscher und Untersenon sowie Bemerkungen über einige verwandte Formen in der Oberkreide. Nova Acta Leopoldina NF, 10 (68): 149-264. 